\title{
Future challenges in colloid and interfacial science
}

\author{
Helmuth Möhwald
}

Received: 9 November 2009/Revised: 1 December 2009 / Accepted: 4 December 2009 / Published online: 6 January 2010

(C) The Author(s) 2009. This article is published with open access at Springerlink.com

\begin{abstract}
This article deals with topics where I expect special future challenges, exemplifying these by experiments out of my own department. One area where I expect large progress also in view of many technical developments in the past concerns the understanding of the structure of fluid interfaces at the atomic level. It is shown by nonlinear optical spectroscopies that the free water surface is ice-like and can be "liquefied" by ion adsorption. X-ray fluorescence from the interface demonstrates that ion binding is very specific which cannot be explained by existing theories. A second major area are nonequilibrium features, and one of the old and new ones here is nucleation and growth. This presentation concentrates on effects produced by ultrasound, a well-defined trigger of gas bubble formation. It exhibits high potential for chemistry at extreme conditions but with a reactor at normal conditions. It has special importance for treatment of surfaces that can be also manipulated via controlled surface energies. A third area will concern complex and smart systems with multiple functions in materials and biosciences. As next generation, I anticipate those with feedback control, and examples on this are self-repairing coatings.
\end{abstract}

Keywords Colloids $\cdot$ Interfacial science $\cdot$ Interfaces

\section{Introduction}

Colloid science deals with entities in size ranges between $1 \mathrm{~nm}$ and some micrometers, due to these small dimensions

Presented at the 44th Assembly of the Kolloid-Gesellschaft on the occasion of receiving the Wolfgang Ostwald award.

H. Möhwald $(\bowtie)$

MPI für Kolloid- und Grenzflächenforschung,

14424 Potsdam-Golm, Germany

e-mail: moehwald@mpikg.mpg.de the surfaces are most important, and thus it is closely linked with interface science. At the lower size limit, it embraces nanosciences which deals with dimensions below $100 \mathrm{~nm}$, although not all scientists in this field have realized how much it relies on classical colloid science. On the other hand the field is defined by a dimension, not by a type of material and it therefore includes inorganic, organic and biomaterials. Thus, it is the basic of many fashionable disciplines like bio-, medical and nanotechnology, and hence will experience a bright future as enabling science. However, one may ask the questions if there are also challenges in the development of colloid and interface science and if there emerged new advances to tackle these challenges. Answers to these questions are "yes", and I will below give examples from work out of my own department. These examples are not at all exhaustive, and I have selected them because of own competence but by no means claiming to cover this broad field. I see major challenges in the following areas:

- Structure and dynamics of fluid interfaces at submolecular resolution.

- Nonequilibrium structures and properties.

- From smart to feed back and remote controlled systems.

\section{Areas of challenges}

Structure and dynamics of fluid interfaces

The last 2 decades have seen a tremendous development of techniques to study interfaces directly and with molecular resolution. These are synchrotron X-ray [1] and Neutron scattering [2], FTIR spectroscopy [3], Nonlinear optical spectroscopies [4] as well as simulations [5]. Concerning molecules or particles at interfaces, the trend has moved from classical insoluble surfactants and phospholipids towards more complex molecules like amphiphilic polymers, peptides, 
proteins and their mixtures as well as nanoparticles. This is understandable from the desire to construct more complex systems and also to answer biomedical questions. On the other hand, there are still burning questions related to most simple interfaces and on these I will present two examples below:

The first concerns the structure of water at the free surface and its influence by ions in the subphase. To conclude on this VIS/IR sum frequency generation (SFG) has been established as a most powerful tool [6, 37]. Applying SFG a high intensity visible and a tuneable IR beam are impinging on a surface, and a beam at the sum frequency may result if the IR beam is in resonance with a vibration. Since this is a second order non-linear effect only species at the interface with a symmetry break are detected. Figure 1 shows a typical spectrum of the water surface with various amounts of $\mathrm{SCN}^{-}$ in the subphase. The bands of the ion free water surface have traditionally been assigned to the free $\mathrm{OH}$ vibration $\left(3700 \mathrm{~cm}^{-1}\right)$, liquid water and "solid" water $\left(3,450 \mathrm{~cm}^{-1}\right.$ and $3,200 \mathrm{~cm}^{-1}$, respectively). Accordingly one realizes that the ice like peak is reduced by ion addition, i.e. that the ion disturbs the hydrogen bonding network in agreement with simulations [7]. Measuring the SFG signal for different polarizations one also deduces that the ion, in this case an anisotropic one, is preferentially oriented at the surface.

There are also many attempts to measure ion distribution at charged interfaces which would be a stringent test of theories. However, estimates for charged monolayers indicate that even for monovalent ions more than $90 \%$ of them are expected within a distance below $5 \AA$ from the surface [8]. Therefore one needs methods with spatial resolution better than $1 \AA$, and this is very difficult to achieve. One way could be to use standing X-ray waves which can be produced via interference at interfaces, but even the most sophisticated attempts have been not convincing in view of this goal [9]. An alternative may be not to dwell on distribution but to measure competitive ion binding. As an example Fig. 2 shows measurements of $\mathrm{X}$-ray fluorescence of different ions near an interface [8]. In this example an evanescent X-ray beam hits the surface exciting ions residing within its penetration depth of about $50 \AA$. The fluorescence of these ions is then detected measuring their concentration. For calibration one may use films with exclusively one ion in the subphase or a higher incidence angle where the bulk concentration is detected. Principally one can also vary the penetration depth via the incidence angle, but in reality this variation is

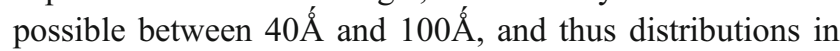
this range, e.g. for polymer brushes, can be resolved. In the specific example of Fig. 2, top, one can distinguish the emission of S (from the surfactant) and of argon (from the gas in the measurement chamber) which may serve for normalization, and the emission of $\mathrm{K}^{+}$and of $\mathrm{Cs}^{+}$. Comparing with the situation of only one ion in the subphase the emission of both ions is reduced by a factor
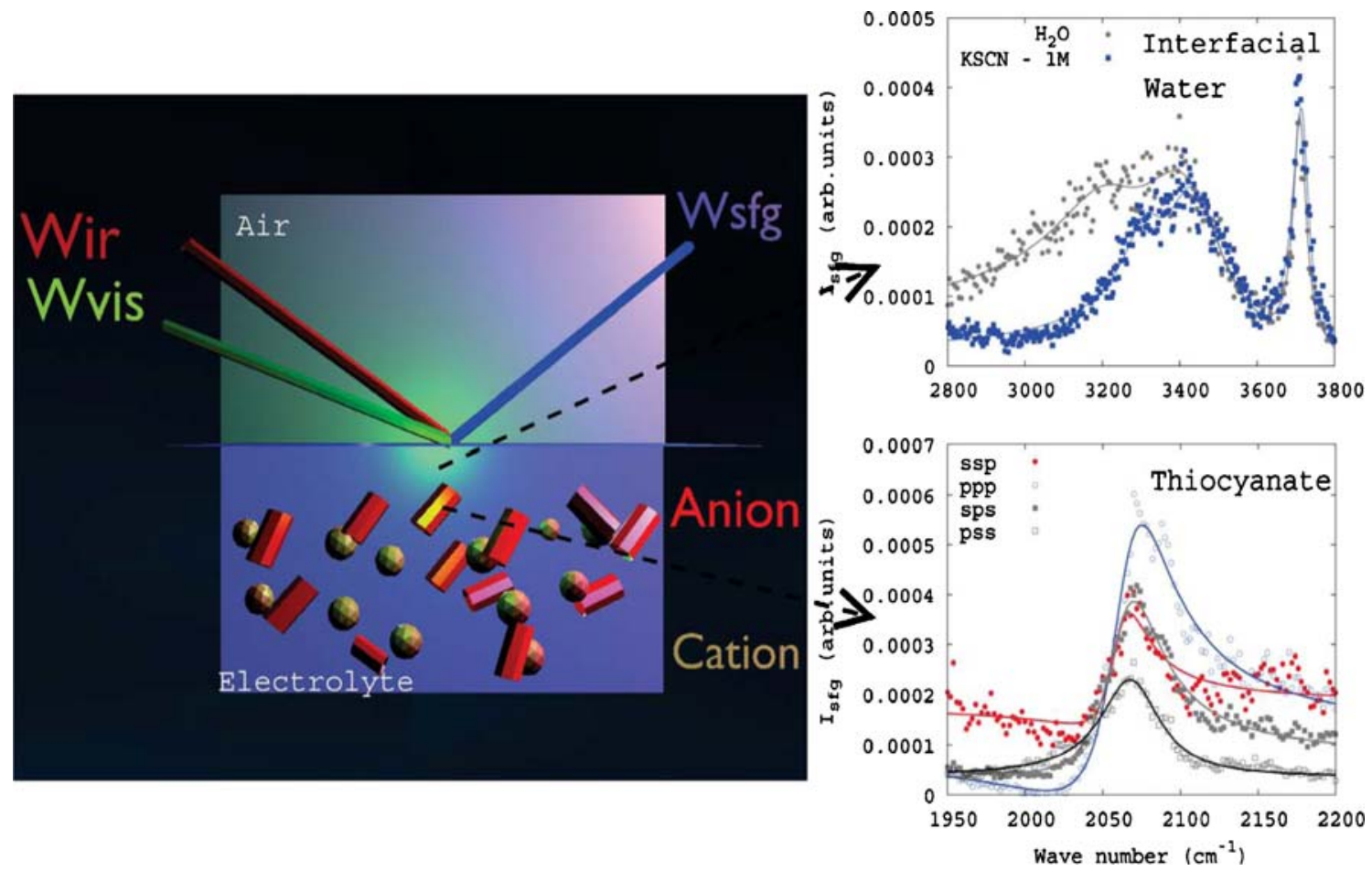

Fig. 1 Left: Sketch of a sum frequency measurement. A visible (Wvis) and an IR (Wir) laser beam impinge on a surface and scattering with the sum frequency Wsfg may result in resonance with Wir. Right top
SFG spectrum of the free water in absence (grey) or presence of KSCN (dark) in the subphase. Right bottom: SFG spectrum of the $\mathrm{SCN}^{-}$anion for different polarization of incoming and reflected beam [6] 


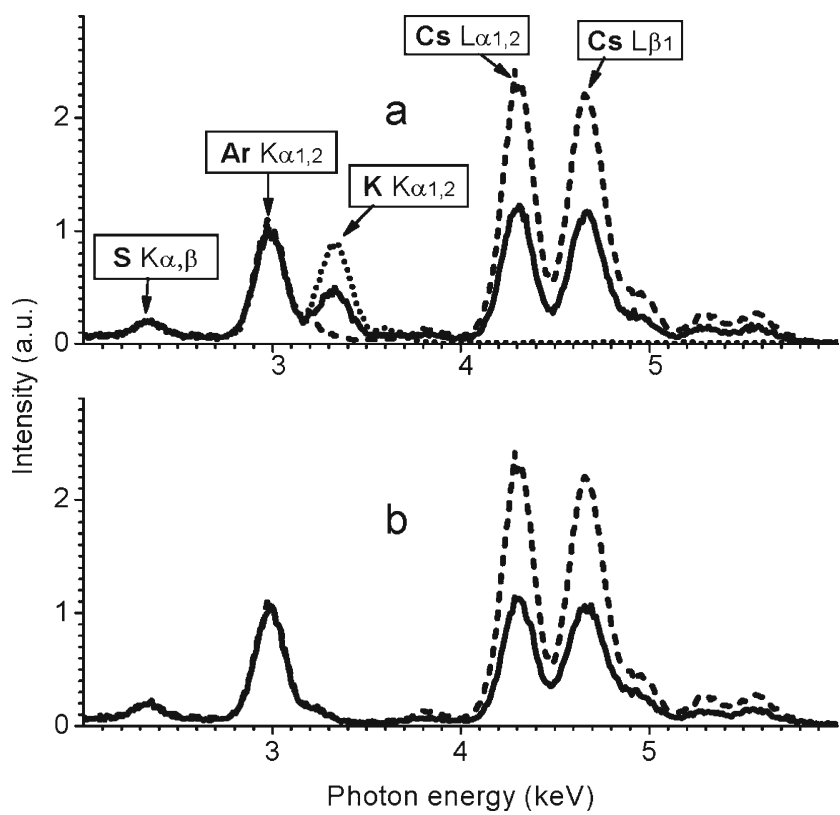

Fig. $2 a$ X-ray fluorescence spectrum after irradiation by an evanescent $\mathrm{X}$-ray beam with peaks of $\mathrm{S}, \mathrm{Ar}, \mathrm{K}$ and $\mathrm{Cs}$ for the solution containing only one type of cation and for a 1:1 mixture (full line). b X-ray fluorescence spectrum for a solution containing only $\mathrm{Li}^{+}$and $\mathrm{C}_{\mathrm{S}}{ }^{+}$and for a $\mathrm{Li}: \mathrm{C}_{\mathrm{S}}=$ 9:1 ratio (full line) [8]

of 2. This lack of ion specifity is expected if the ions do not bind at all or with the same strength. A qualitatively different behaviour is, however, expected comparing $\mathrm{Li}^{+}$ and $\mathrm{Cs}^{+}$(Fig. 2, bottom). There, an excess of $\mathrm{Li}^{+} / \mathrm{Cs}^{+}$of 9:1 is apparently necessary to achieve equal binding. These studies can be extended to many different ions, but they need a dedicated set-up at a synchroton. They will provide very important information on ion-specific binding including the Hofmeister effect [10], but will require dedicated theoretical simulations to achieve an understanding.

In view of the rapid progress concerning X-ray sources including free electron lasers one may ask how this will affect science with fluid interfaces. I am convinced that by these X-ray sources other techniques, previously developed for hard surfaces in vacuum, will become available like photoelectron spectroscopy or extended X-ray fine structure analysis with glancing X-rays. The latter could shed light on the local environment of binding ions, an important question concerning the above example. Also more refined analysis of two-dimensional arrays of polymers, peptides, proteins and nanoparticles will be possible. I am, however, sceptical on the use of the most brilliant X-ray sources. They cause severe sample damage within some fsec, and the questions will be which information can be gathered within this time frame.

Nonequilibrium structures and properties

Many colloidal systems are kinetically hindered to minimize their surface energy and thus are used in a nonequilibrium state. Prominent classical areas are emulsions and dispersions but also the field of nucleation and growth has encountered much interest at least since the times of Wolfgang Ostwald [29]. Although being far away from a quantitative understanding it encounters at present a boom in view of preparation of nanoparticles of defined size and shape and their aggregation $[11,36]$. I will spare here examples of my department on this topic $[12,34]$ but concentrate on the field of sonochemistry which sounds exotic and is rather difficult to study quantitatively. On the other hand it is very important and many scientists have used ultrasound to clean a surface or to disperse a sample by this process.

If a liquid is exposed to ultrasound gas bubbles may be nucleated and grown (cavitation) and the later collapse of the bubble converts the surface energy into mechanical energy and heat. Thus temperatures above $5.000 \mathrm{~K}$ and pressures above $1.000 \mathrm{~atm}$ result for short times $(<\mu \mathrm{s})$ and very local $(<\mu \mathrm{m})$ [13]. Due to extreme cooling rates $\left(\sim 10^{10} \mathrm{~K} / \mathrm{s}\right)$ structures can thus be formed far away from equilibrium. Examples are composites of immiscible elements, sintering of particles, cross-linking of polymers and proteins, flotation and selective dispersion from solids.

Hence one can perform high temperature and pressure chemistry with a reactor at normal conditions, but the main problem arises to control nonequilibrium processes locally and in short time. As an example of using high pressures Fig. 3 shows electron micrographs of clays sonochemically treated in the presence of $\mathrm{Au}$ nanoparticles and surfactant [14]. Here ultrasound creates defects into which the nanoparticles and surfactants are injected. The surfactant causes exfoliation, and hence a large amount $(\sim 5 \%)$ of particles can be stably inserted. This system serves as a model of a catalyst carrier, and in the process, compared to conventional procedures, the incorporation time is reduced from days to less than an hour, i.e. by about a factor 100 .

In the example of Fig. 3, the cavitation bubble is formed in bulk with clay and Au nanoparticles concentrating at the bubble surface. The process may be easier to describe, at least theoretically, if one considers sonochemistry at surfaces. There, one expects the situation of homogeneous versus heterogeneous nucleation (Fig. 4) [15] and the latter should be favoured, the amount depending on the surface energy of the solid. Consequently preparing a surface with hydrophobic and hydrophilic patterns one expects preferential bubble nucleation on the hydrophobic areas. This can indeed be verified (Belova V, Gorin D, Shchukin DG, Mohwald $\mathrm{H}$., to be published; Fig. 4). Preparing by stamping an $\mathrm{Al}_{2} \mathrm{O}_{3}$ surface with hydrophilic spots in a hydrophobic laterally continuous area one can clearly realize the influence of the ultrasound causing nm-sized corrugations only on the hydrophobic areas. Although in this case a measurement of nucleation rates and a theoretical relation between nucleation rate, contact angle 


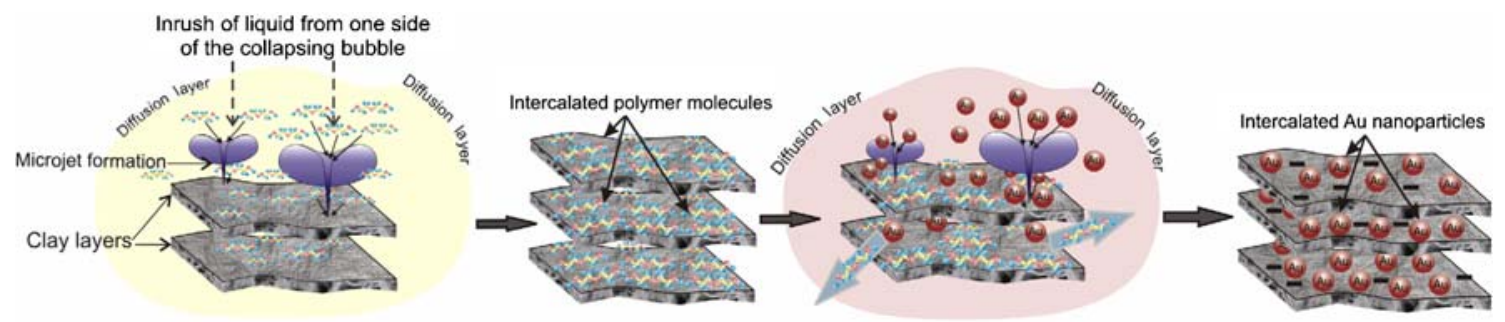

First sonication

Second sonication
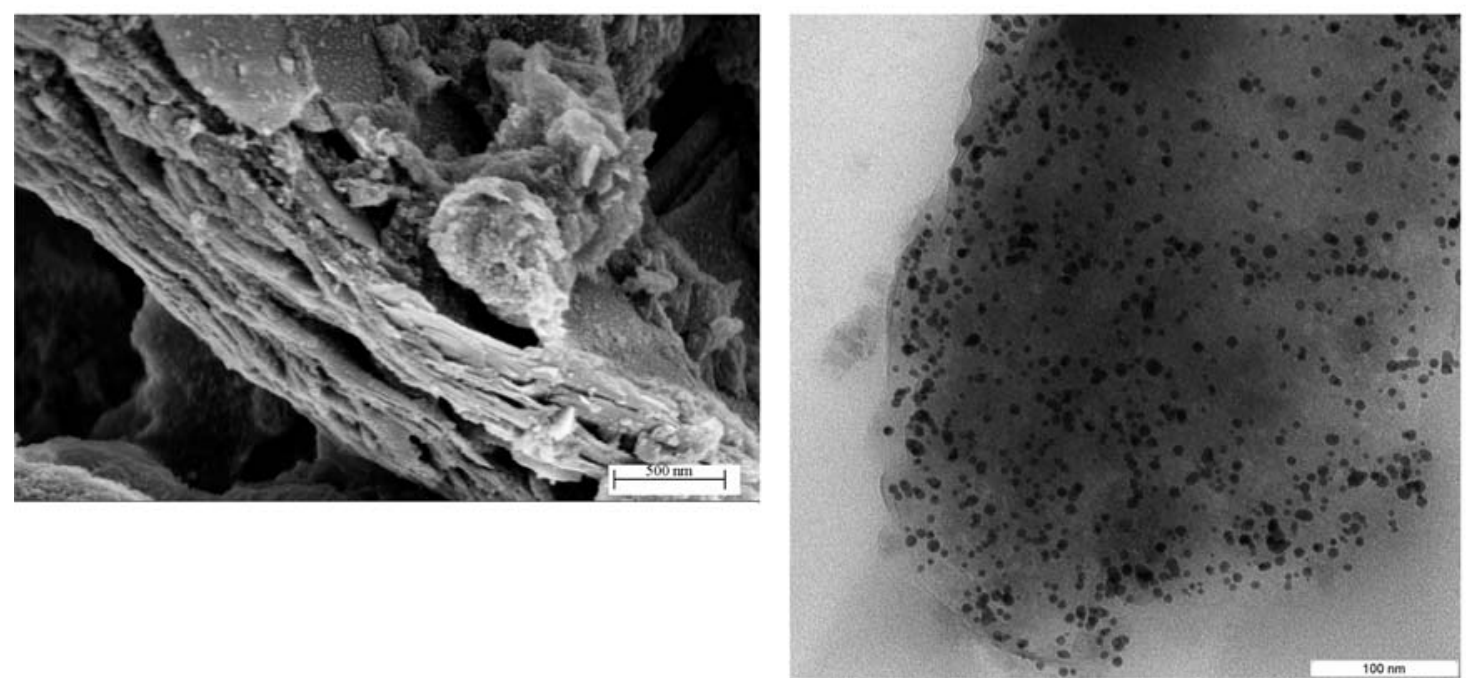

Fig. 3 Top: Scheme of ultrasonic exfoliation of clays by tensides (left) and following insertion of Au nanoparticles (right). Bottom: SEM of clay microparticles (left) and TEM of Au nanoparticles on clay sheets [14]

Fig. 4 Top left: Bubble nucleation and growth in bulk and at a surface. Whereas in the first case the surface tension 6 of the liquid is most important, in the second case also the interfacial tension $\sigma_{\mathrm{S}}$ of the solid and the contact angles are relevant, the gas pressures $\mathrm{P}$ and chemical potentials $\mu$ in both phases being equal. Top right: Sketch of a pattern and the molecule used for hydrophobization. Bottom: SEM image at different magnification of an Al surface after $10 \mathrm{~min}$ of ultrasonication
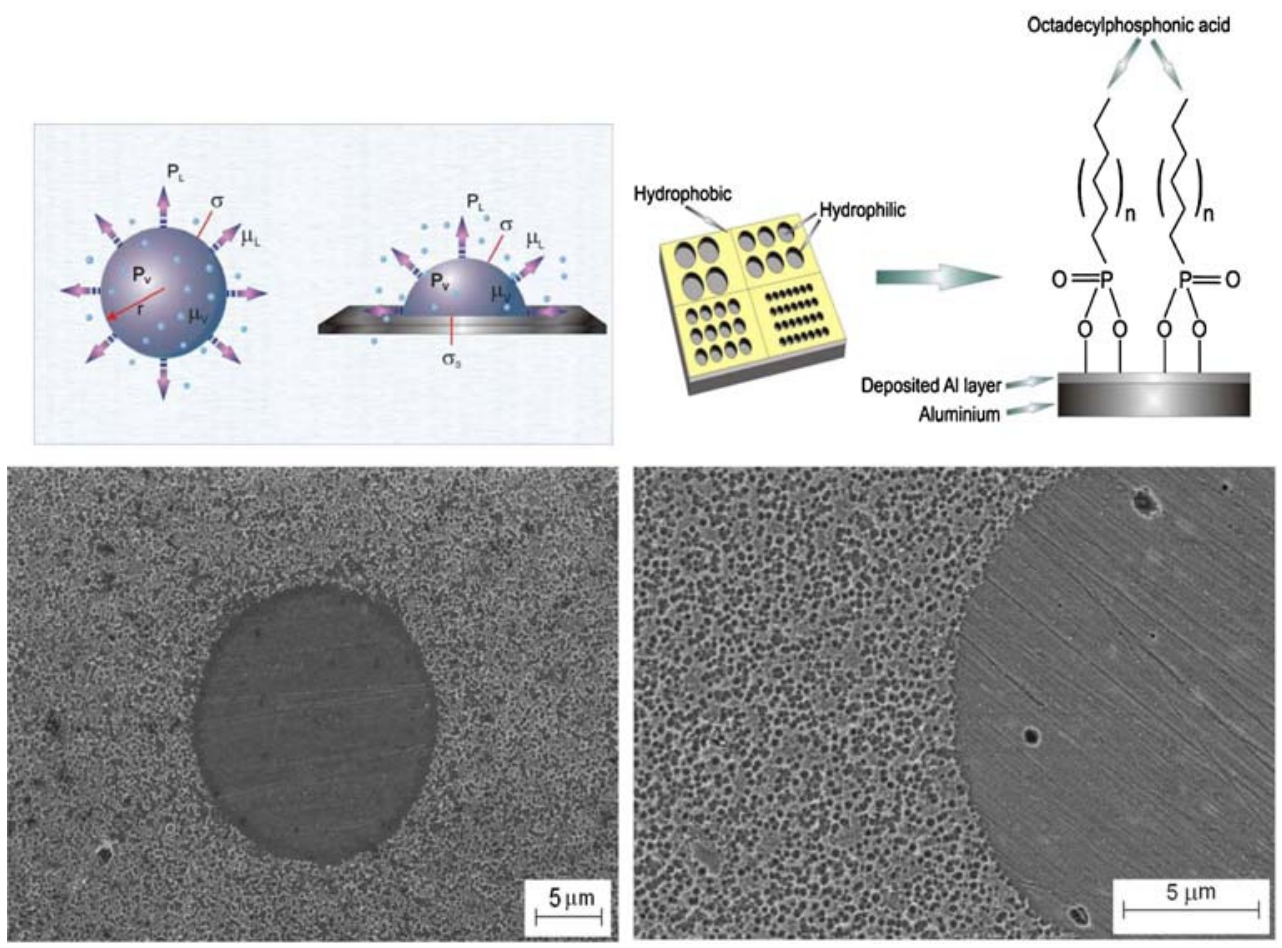
and ultrasonic power are still pending, I expect this is the beginning of a field with large potential: Varying and measuring contact angles one should be able to control nucleation, varying the sizes of hydrophobic areas one should learn about critical radii, varying their shape one may also produce peculiar bubble shapes [16]. With ultrasound as a reproducible trigger and patterning one can also define time and space of nucleation enabling direct measurements of nucleation and growth via optical scattering and imaging. On the application side one can use the high definition to prepare and/or to use patterned surfaces and control their selective dispersion as well as to use the process for selective cleaning without destruction.

From smart to feed back and remote controlled systems

Ultrasound has also revealed to be an important tool in the design of feedback active coatings as is shown by means of the example of Fig. 5. There use is made of the fact that ultrasound causes defined surface corrugations (Fig. 5a), and predominantly by FTIR-spectroscopy one may show that the surface is completely oxidized [17]. This, in contrast to the untreated surface, enables a complete surface coating by the layer-by-layer technique [18]. This technique is very versatile enabling incorporation of many different functional molecules. It can also be extended to prepare containers with walls defined as precise as multilayers but containing large amounts of functional molecules. Of special importance here is the fact that in many cases stability and properties are determined by electrostatic interactions. These in turn can be modulated via $\mathrm{pH}$ [19], salt or electrochemical potential [20], and this is made use of in designing corrosion protective coatings. Figure $5 b$ shows even macroscopically that a surface coated suitably is protected against visible corrosion in contrast to an unprotected one [20].

The principles of this protection mechanism can be understood and also quantified by means of the scheme and results in Fig. 6: If a corrosion pit forms its local potential or $\mathrm{pH}$ differs from that of the majority of the surface. This then affects the electrical balance within the coating, in consequence increasing its permeability and releasing bound or encapsulated (in dispersed nanocontainers) corrosion inhibitors. They then anneal the defect, and we therefore have a self-repairing coating [21]. The action of this mechanism can be followed by scratching the coating and locally following the corrosion
Fig. 5 Top: Sketch of a bubble in bulk and on a hydrophilic (Al) surface. Bottom left: SEM image of untreated (a) and sonochemically treated $\mathrm{Al}(\mathbf{b})$. Bottom right: Optical micrograph of untreated (top) and treated (middle) $\mathrm{Al}$ after $24 \mathrm{~h}$ in salt solution. Right lower corner: Sketch of the coating of the ultrasonicated surface by the layer-by-layer technique with corrosion inhibitor inside [17]
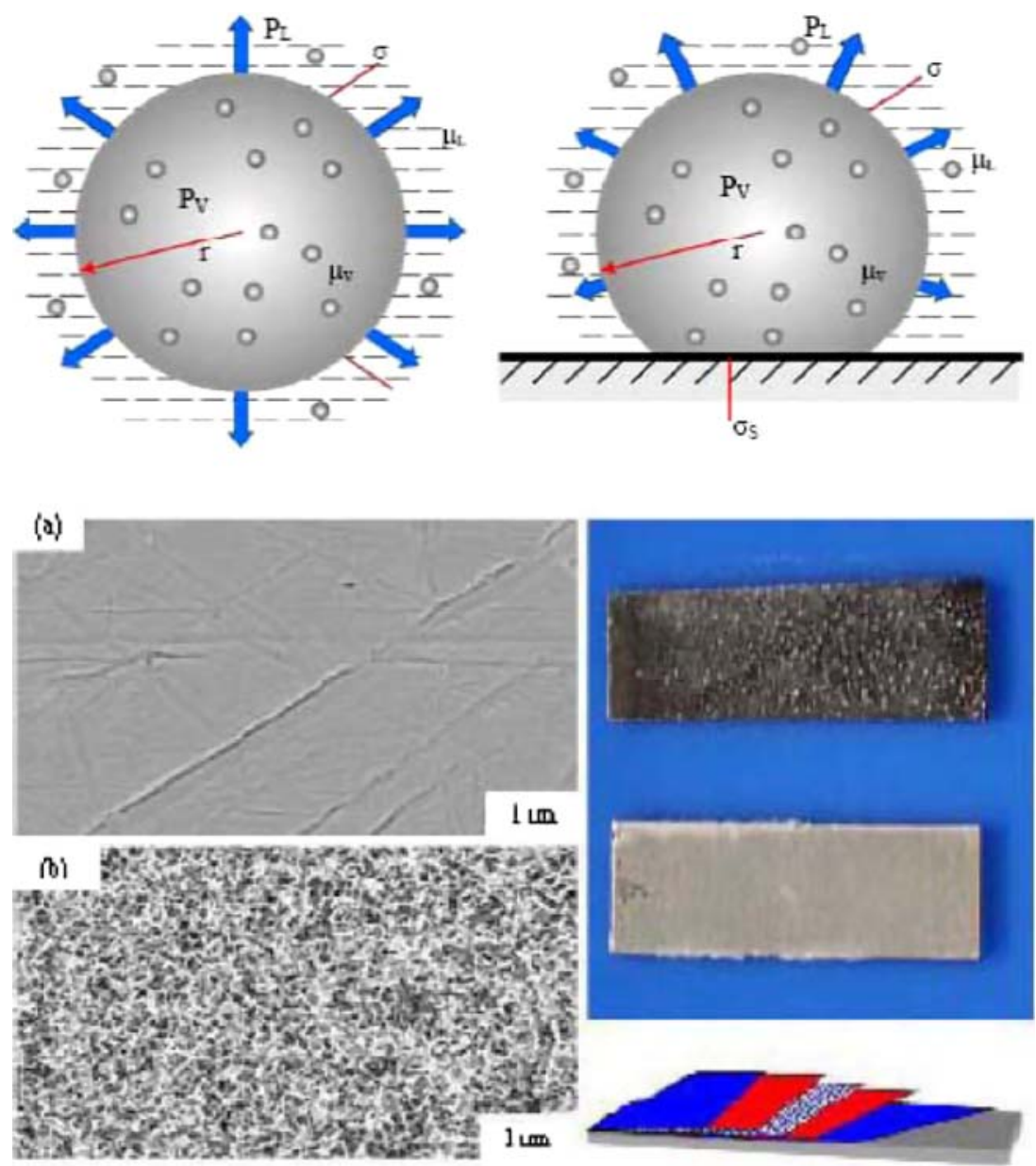
Fig. 6 Top left: Concept of self-repairing coating: Nanocontainers are opening near a defect due to a different local $\mathrm{pH}$ or electrochemical potential and thus release a corrosion inhibitor. This then anneals the defect. Bottom left: Possible realization: $\mathrm{SiO}_{2}$ nanoparticles are coated by multilayers of (polyethyleneimine $(P E I)$ and polystyrenesulfonic acid (PSS) and embedded into a $\mathrm{ZrO}_{2} / \mathrm{SiO}_{2}$ sol/ gel coating on an $\mathrm{Al}$ alloy surface technically used. Top right: Laterally resolved corrosion current in $\mu \mathrm{A} \mathrm{cm} \mathrm{cm}^{-2}$ for an unprotected surface after indicated times in salt solution (a) following a scratch on the surface seen in the optical micrograph (b). Bottom right: Time and space dependent corrosion current for a surface protected by an inhibitor containing layer (a) following a scratch optically visualized (b)
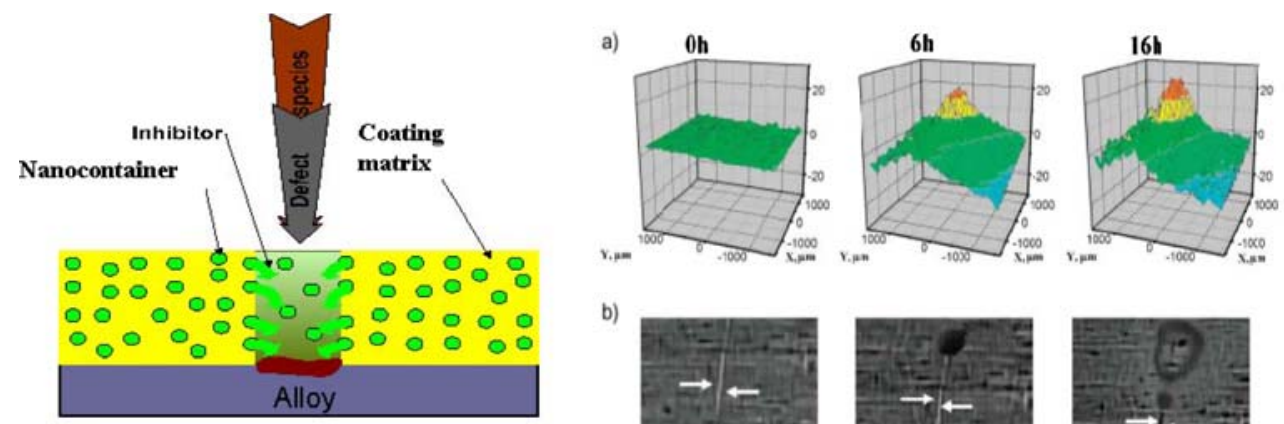

b)
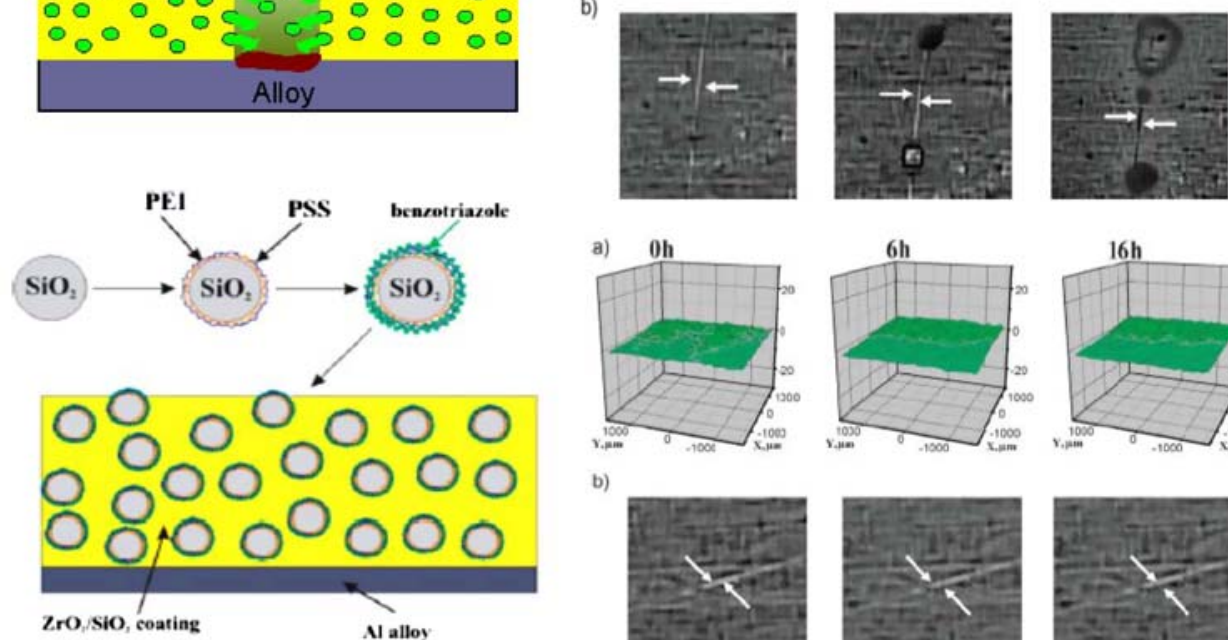
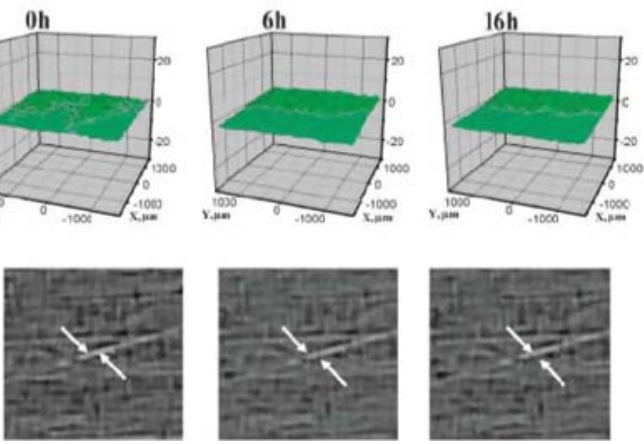

current by the scanning vibrating electrode technique. One realizes in Fig. 7 that the corrosion current increases for the unprotected surface but it decays with time for the protected one due to the inhibitor action. In addition there may be a change in local mobility in the polyelectrolyte multilayer enabling molecular movement to seal the defected coating.

In the above example we have made use of one of the many present developments of so-called smart materials, i.e. materials that respond to the environment [31]. These are the base elements of more sophisticated devices that, analogous to many regulating and regulated systems are connected as a positive or negative feedback [21]. Feedbacks are typically used in nature or technology to maintain a certain concentration, potential or mechanical force on a desired level and uses are maximum amplification for a triode, fast response for a muscle or delivery of stored chemical energy on demand. Here the prime questions will not concern molecular mechanisms but the interplay and coupling of different responsive elements.

I expect that another important area in the future will be the remote control of processes via external fields, electromagnetical, optical, acoustical or thermal ones. For demonstration, I selected an example of own work towards biosciences [22]. The base elements here are:

1. Polyelectrolyte multilayer capsules [30], which are known to be at high temperature very soft and permeable also for macromolecules, are glassy and virtually impermeable at low temperature [23]. The transition between high and low temperature can be described like a glass transition, and the corresponding temperature may be tuned via $\mathrm{pH}$, salt and type of polymer between room temperature and $90^{\circ} \mathrm{C}$.

2. Metallic inorganic nanoparticles can be incorporated into the multilayers. Via aggregation, preparation of rods or core/shell geometry they can be made to strongly absorb near IR-light [24, 25]. This light is then converted into heat and one can show that with a low power IR diode one can increase the temperature of the particle by some $10 \mathrm{~K}$ whereas the temperature may be kept below $35^{\circ} \mathrm{C}$ some $10 \mathrm{~nm}$ apart. Therefore one can create locally hot spots with permeability for large molecules controlled spatially and temporally via the IR laser diode.

3. By consecutively heating and shrinking of the capsules in a solution of a signal peptide the latter can be encapsulated and these capsules can be brought into a cell by electroporation [22]. This method can be used for virtually any type of cells (even those which don't exhibit phagocytosis), although other cells like macrophages or various cancer cells would incorporate these capsules spontaneously [26].

4. Since the capsules are not biodegradable one can wait for some hours till the cells have recovered from the harsh electroporation treatment. Then one may select a specific capsule and location and open it by IR light, the process we call optoporation [35].

5. Intracellular delivery and release can be used for investigation of a receptor-mediated antigen presentation [27] - a process of fundamental importance in 

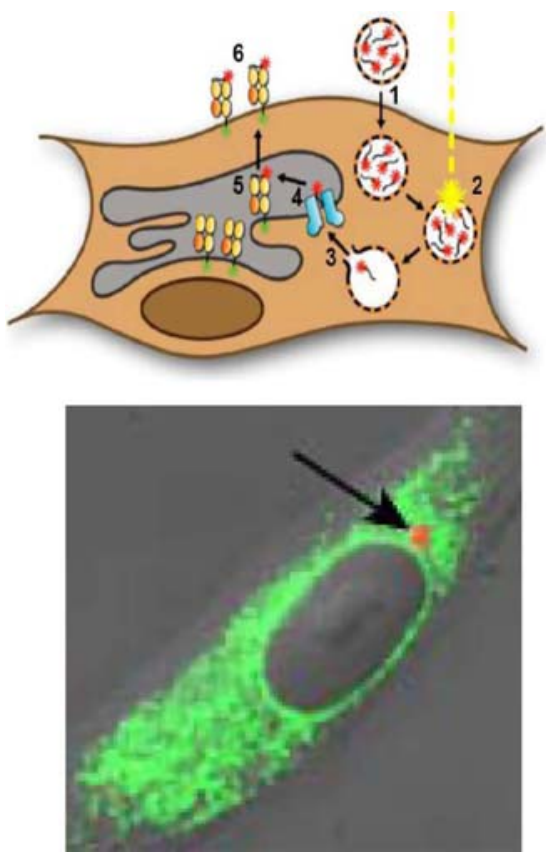

Fig. 7 Top: Scheme describing immunological response under study. A capsule containing the signal peptide SIINFEKL is brought into a mammalian cell (Vero-cells) and opened there by IR-light $(1-3)$. The released peptide is bound to the receptor MHC Class I proteins (4) forming the complex, and is finally transported to the cell surface (5). There it exhibits the surface presentation (6). Bottom: Confocal fluorescence micrograph showing the cytosol (green), the nucleus (dark ellipse) and a capsule (red dot) [22]

immunology. The idea of these experiments consists in bringing a microcapsule filled with peptides into a mammalian cell. Then one can follow the binding of the peptide to a receptor, trace its intracellular transport, and eventually use the corresponding antibodies to investigate the surface presentation. Approaches typically used for assessing this and, it is fair to say, other biological processes are rather descriptive, so further developments are expected in using math for quantification [22].

Figure 7 (top) presents a model of the process and the expected response, while Fig. 7 (bottom) shows that the encapsulated peptide (red) can be brought near the cell nucleus (dark ellipse). According to our model, the released signal peptide should be transferred via several compartments, bind to a receptor and form the complex which should be transported to the cell surface, Fig. 7 (top) [32, 33]. A fine detail of this experiment is the surface binding of the antibody which is specific to the complex but which is added from outside of the cell. The binding manifests visibility of the cell under study by T-cells - the hall-mark of the immune system response [22]. The question now arises if this model is correct and at which time scales the processes occur.

To answer the above question Fig. 8 shows time dependent fluorescence micrographs on the distribution of the peptide (SINFEKL-TAMRA) and of the receptor MHC class I. Measuring the intensity at the center of the cell and at the edge one can thus quantify the transport of the receptor to the surface (right in Fig. 8), and via different labelling one can also verify the coupling of an antibody from outside. This confirms that the suggested model is correct and that the corresponding processes last on the order of hours [22].

In this example as well as in the previous one the task is not to answer fundamentally new questions although this is possible too, but to look into the interplay of multiple components, the problems coming from disciplines like materials science, medicine and biology.

In this respect colloid and interface science may become a helper science which I do not mean negative since this help will be most important and can spread into many disciplines.

\section{Conclusions and outlook}

In this brief contribution I intended to point into three different areas where I believe colloid and interface science to develop in the future. I have selected examples from my own environment and apologize to those colleagues whom I did not cite nor mention their directions. The field is very
Fig. 8 Left: Fluorescence micrograph of the distribution of a green labelled receptor (top row) as a function of time (from left $0,10,20 \mathrm{~h}$ ) and of the signal peptide (red labelled, bottom row). To quantify the increasing receptor concentration at the cell surface the intensities at the edge are related to those at the center (right in Fig. 8). The scale bar corresponds to $50 \mu \mathrm{m}$ [22]
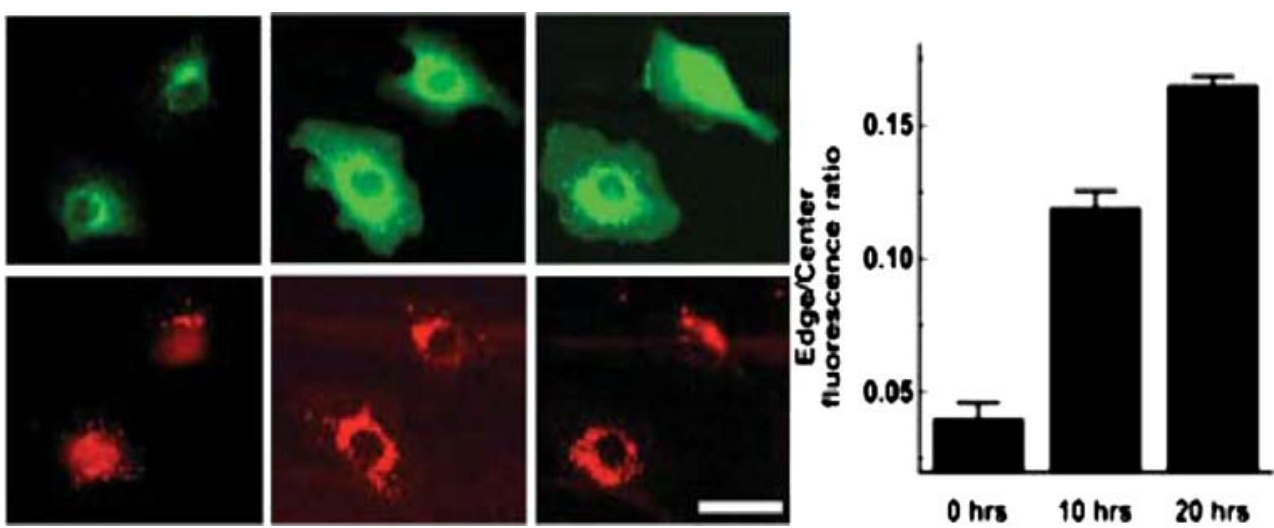
broad because it is not confined to any type of material and building up of hierarchical structures and functions is a challenge depending also on the types of material. Among these materials, supramolecular systems are especially promising because the interplay of their weak interactions enables a variety of hierarchical structures and functions [28].

There is also a trend to answer more biological questions, and one should be aware that there is no qualitative difference between synthetic and biological molecules. On the other hand biological techniques are now at hand to synthesize well-defined and interesting molecules which may become optimum models for our science, e.g. carbohydrates with most defined structures as recognition elements and as hydrogels. Therefore our science may not only help other disciplines but these may be also useful for us. In any case I am optimistic that colloid science has a bright future on one hand as helper science on the other hand it provides still many challenging fundamental questions.

Acknowledgement During my career I have profited and learnt from the enthusiastic, motivated and skillfull contributions of many students, postdocs, group leaders and colleagues. I am especially grateful to those former or present co-workers from whom I could take examples to present here as figures, notably G. Brezesinski, $\mathrm{H}$. Motschmann, D.G. Shchukin, V. Belova, D.V. Andreeva, D. Fix and A.G. Skirtach. I do not list the contributions of the many funding agencies, because they only pushed us to work more and more. Still I hope they are satisfied with our output.

Open Access This article is distributed under the terms of the Creative Commons Attribution Noncommercial License which permits any noncommercial use, distribution, and reproduction in any medium, provided the original author(s) and source are credited.

\section{References}

1. Kjaer K, Als-Nielsen J, Helm CA, Laxhuber LA, Möhwald H (1987) Ordering in lipid monolayers studied by synchroton X-ray diffraction and fluorescence microscopy. Phys Rev Lett 58:2224

2. Penfold J, Thomas RK (1990) The application of the specular reflection of neutrons to the study of surfaces and interfaces. J Phys, Condens Matter 2:1369-1412

3. Dluhy RA, Cameron DG, Mantsch HH et al (1983) Fouriertransform infrared spectroscopic studies of the effect of calciumions on phosphatidylserine. Biochemistry 22:6318-6325

4. Du Q, Superfine R, Freysze E et al (1993) Vibrational spectroscopy of water at the vapor water interface. Phys Rev Lett 70:2313-2316

5. Hautmann J, Klein ML (1990) J Chem Phys 93:7483-7492

6. Viswanath P, Motschmann H (2008) Effect of interfacial presence of oriented thiocyanate on water structure. J Phys Chem C 112:2099-2103

7. Jungwirth P, Tobias DJ (2001) Molecular structure of salt solutions: A new view of the interface with implications for heterogeneous atmospheric chemistry. J Phys Chem B 105:10468-10472
8. Shapovalov VL, Brezesinski G (2006) Breakdown of the GouyChapman model for highly charged Langmuir monolayers: counterion size effect. J Phys Chem B 110:10032-10040

9. Schollmeyer H, Guenoun P, Daillant J, Klitzing RV, Novikov DV et al (2007) Ion distribution in polyelectrolyte multilayers with standingwave X-ray fluorescence. J Phys Chem B 111:4036-4042

10. Bostrom M, Kunz W, Ninham BW (2005) Hofmeister effects in surface tension of aqueous electrolyte solution. Langmuir 21:2619-2623

11. Peng XG, Manna L, Yang WD et al (2000) Shape control of CdSe nanocrystals. Nature 404:59-61

12. Zhang H, Wang DY, Yang B, Möhwald H (2006) Manipulation of aqueous growth of CdTe nanocrystals to fabricate colloidally stable one-dimensional nanostructures. J Am Chem Soc 128:10171-10180

13. Raleigh J (1917) Proc R Soc (London) A 93:148

14. Belova V, Mohwald H, Schukin DG (2008) Sonochemical intercalation of preformed gold nanoparticles into multilayered clays. Langmuir 24:9747-9753

15. Volmer M, Schultze W (1931) Condensation of crystals. Z Phys Chem, A Chem Thermodyn Kinet Elektrochem Eigenschlehre 156:1-22

16. Lipowsky R, Brinkmann M, R Dimova et al (2005) Droplets, bubbles, and vesicles at chemically structured surfaces. J Phys, Condens Matter 17:S537-S558

17. Andreeva DV, Fix D, Mohwald $\mathrm{H}$ et al (2008) Self-healing anticorrosion coatings based on pH-sensitive polyelectrolyte/ inhibitor sandwichlike nanostructures. Adv Mater 20:2789

18. Decher G, Hong JD (1991) Buildup of ultrathin multilayer films by a self-assemly process. 1. Consecutive Adsorption of Anionic and Cationic Bipolar Amphiphiles on Charged Surfaces. Conference Information: 3rd European Conf on Organized Organic Thin Films (ECOF 90), Date: Oct 11-13, 1990 Johannes Gutenberg Univ Mainz FED REP GER, Makromolekulare Chemie-Macromolecular Symposia 46, 321-327

19. Sukhorukov GB, Antipov AA, Voigt A, Donath E, Möhwald H (2001) pH-controlled macromolecule encapsulation in and release from polyelectrolyte multilayer nanocapsules. Macromol Rapid Commun 22:44-46

20. Shchukin DG, Köhler K, Möhwald H (2006) Microcontainers with electrochemically reversible permeability. J Am Chem Soc 128:4560-4561

21. Zheludkevich ML, Shchukin D, Yasakau KA, Möhwald H, Ferreira MGS (2007) Anticorrosion coatings with self-healing effect based on nanocontainers impregnated with corrosion inhibitor. Chem Mater 19(3):402-411

22. Palankar R, Skirtach AG, Kreft O et al (2009) Controlled intracellular release of peptides from microcapsules enhances antigen presentation on MHC class I molecules. Small 5:2168-2176

23. Kohler K, Sukhorukov GB (2007) Heat treatment of polyelectrolyte multilayer capsules: a versatile method for encapsulation. Adv Funct Mater 17:2053-2061

24. Skirtach AG, Karageorgiev P, Bedard MF et al (2008) Reversibly permeable nanomembranes of polymeric microcapsules. J Am Chem Soc 130:11572

25. Skirtach AG, Dejugnat C, Braun D, Susha AS, Rogac AL, Parak J, Möhwald H, Sukhorukov GB (2005) The role of metal nanoparticles in remote release of encapsulated matrials. Nano Lett 5:1371-137

26. Radt B, Smith TA, Caruso F (2004) Optically addressable nanostructured capsules. Adv Mater 16:2184

27. Townsend A, Bodmer H (1989) Antigen recognition by class-I restricted lymphocyte-T. Annu Rev Immunol 7:601-624

28. Cohen Stuart MA (2008) Supramolecular perspectives in colloid science. Coll Polym Sci 286:855-864

29. Ostwald W (1927) The theory of the precipitate rule. KolloidZeitschrift 43:249-267 
30. Donath E, Sukhorukov GB, Caruso F, Davis SA, Möhwald H (1998) Novel hollow polymer shells by colloid-templated assembly of polyelectrolytes. Angew Chem 37:2201-2205

31. Stieger M, Richtering W, Pedersen JS et al (2004) Small-angle neutron scattering study of structural changes in temperature sensitive microgel colloids. J Chem Phys 120:6197-6206

32. Bjorkman PJ, Saper MA, Samraoui B et al (1987) Structure of the human class-I histocompatibility antigen, HLA-A2. Nature 329: 506-512

33. Garstka M, Borchert B, Al-Balushi M et al (2007) Peptidereceptive major histocompatibility complex class I molecules cycle between endoplasmic reticulum and cis-golgi in wild-type lymphocytes. J Biol Chem 282:30680-30690
34. Radziuk D, Shchukin D, Mohwald H (2008) Sonochemical design of engineered gold-silver nanoparticles. J Phys Chem C 112: 2462-2468

35. Skirtach AG, Javier AM, Kreft O, Köhler K, Alberola AP, Möhwald H, Parak WJ, Sukhorukov GB (2006) Laser-induced release of encapsulated materials inside living cells. Angew Chem 45:4612-4617

36. van Embden J, Sader JE, Davidson M et al (2009) Evolution of colloidal nanocrystals: theory and modeling of their nucleation and growth. J Phys Chem C 113:16342-16355

37. Viswanath P, Motschmann H (2007) Oriented thiocyanate anions at the air-electrolyte interface and its implications on interfacial water-a vibrational sum frequency spectroscopy study. J Phys Chem C 111:4484-4486 